\title{
Effects of dietary chitosan nanoparticles on serum lipid concentration in hyperlipidemic rats induced by high-fat diet
}

\author{
Mohamed A. Abd-Elhakeem ${ }^{*}$, Nourhan Farag and Marina Maurice \\ College of Biotechnology, Misr University for Science and Technology, Giza, Egypt
}

\section{A R T ICLE INFO}

Article history:

Received 06 September 2016

Accepted 17 October 2016

Keywords:

Ginkgo biloba;

Inflammation;

Oxidative stress;

Renal carcinogenesis;

Caspase 3.

\begin{abstract}
A B S T R A C T
This study was performed to investigate the hypolipidemic effect of oral administration of chitosan nanoparticles in rats fed with fat -rich diet. Chitosan nanoparticles (CHNPs) were prepared with ionotropic gelation. The obtained nanoparticles were spherical in shape and had a smooth surface with size range between 40 and $60 \mathrm{~nm}$. Forty Rats were equally separated into four groups, a normal diet group (ND), a high fat diet group (HFD), a chitosan powder group (CHP) and chitosan nanoparticles group (CHNPs).Lipids profile including total cholesterol (TC), triglycerides (TG), high-density lipoprotein cholesterol (HDLC) and low-density lipoprotein cholesterol (LDL-C) were analyzed by routine biochemical analyses. Body weight gain, TC, TG and LDL-C were significantly decreased inboth CHP and CHNPs groups than that in the HFD group. Although no significant difference between CHP and CHNPs groups, CHNPs are seemed to have better effect on both body weight gaining and serum lipids concentration.
\end{abstract}

\section{Introduction}

Consumption of fast food increases rapidly throughout our society causing a significant disruption of serum lipids profile ${ }^{[1]}$. These lipid imbalances which known as dyslipidemia is considered one of the major factor for cardiovascular disea ${ }^{[2]}$. Dyslipidemia is characterized by increased fasting concentrations of total cholesterol (TC), triglycerides (TG), and low-density lipoprotein cholesterol (LDL), in conjunction with decreased concentrations of high-density lipoprotein cholesterol (HDL) ${ }^{[3]}$. In recent years, many reports have focused on how to decrease serum lipid concentrations and the absorption of fat in the intestinal tract. Dietary nonnutritive substances such as pectin, psyllium and chitosan have showed some potent hypolipidemic effect [4,5]

Chitosan is a natural cationic polysaccharide obtained from various organisms, including the exoskeleton of crustaceans, some insects and the cell walls of some fungi ${ }^{[6]}$. Due toits biocompatibility, biodegradability and nontoxicity, chitosan has been extensively used in biomedical and pharmaceutical formulations ${ }^{[7-8]}$.

Chitosan contains amino group that bear a positive charge in acidic medium. So, chitosan able to bind negatively charged substrates such aslipids and bile acids [9]. Many previous studies revealed that the ingestion of chitosan has a valuable lowering effect on

* Corresponding author.

E-mail address: mohamed.abdelhakim@must.edu.eg serum lipids both in humans and animals [I0-12]. Furthermore, some trials have suggested that chitosan may decrease body weight ${ }^{[13]}$. These results were related to long-term, high-dose chitosan supplementation. In addition, nausea and constipation have commonly occurred at higher doses ${ }^{[\mathbf{1 4}, \mathbf{1 5}]}$. Generally, as the weight is fixed, the smaller the particle size, the bigger in the total surface area. CHNPs possess very finer particle size, which may help adsorption of more lipids. So, this work aims to investigate the effect of CHNPs as a dietary supplement on serum lipids in experimental rats.

\section{Materials and methods}

Shrimp shells were obtained from local market, chitosan capsules (chitocal) were purchased from local market. TC, TG, HDL-C kits were purchased from Biodiagnostic Co. (Egypt). Hydrochloric acid, sodium hydroxide, acetic acid and sodium tripoly phosphate (TPP) were purchased from LOBA (India), all reagents were of analytical grade and used without further purification.

\section{Preparation of chitosan nanoparticles}

The chitosan nanoparticles (CHNPs) were prepared as described in our previous work ${ }^{[16]}$. Briefly, $200 \mathrm{~g}$ grounded dry shrimp shells were soaked in 1L of $1 \%$ $\mathrm{HCl}$ for 24 hours. The obtained material (chitin) was washed by distilled water several times then soaked in $1 \mathrm{~L}$ of $2 \% \mathrm{NaOH}$ solution overnight to obtain chitin. Finally, the chitosan was obtained via boiling the dry chitin powder in $50 \% \mathrm{NaOH}$ solution for 2 hours. The 
remaining creamy white flakes was collected by filtration and air dried.

The chitosan flakes were transformed into nanoparticles via the TPP ionic gelation mechanism. Briefly, a chitosan solution was prepared by dissolving chitosan in $1 \%(\mathrm{w} / \mathrm{v})$ acetic acid solution until the solution was transparent. TPP $(0.5 \mathrm{mg} / \mathrm{ml})$ was dissolved in deionized water. The chitosan solution was mixed with an equal volume of TPP. The formed nanoparticles were separated by centrifugation at $20,000 \mathrm{~g}$ at $14^{\circ} \mathrm{C}$ for 30 minutes then freeze-dried. The obtained CHNPs were stored at $4{ }^{\circ} \mathrm{C}^{[16]}$.

\section{Characterization of prepared chitosan}

The infrared spectrum of chitosan was recorded with a fourier-transform infrared (FTIR) spectroscopy analyzer (Model JASCO FTIR-6100) within the scanning range 4000-400 $\mathrm{cm}^{-1}$, while the morphological examination was performed by TEM microscope (JEOL JAM-2100HR-EM) in the central lab of National Research Center (NRC).

\section{High- fat diet}

The basic rats $\operatorname{diet}(20.0 \%$ total proteins, $4.3 \%$ total fats, $4.8 \%$ total fibers, $9.5 \%$ moisture, $6.0 \%$ ash) were mechanically mixed with equal amounts of cooking oil and animal fats (obtained from local market) to raise the total fat content up to $30 \%(\mathrm{w} / \mathrm{w})$.

\section{Experimental design}

Forty male Sprague-Dawley rats (weight $=120 \pm 12 \mathrm{~g}$; age $=8$ weeks) were purchased and housed in animal house (Faculty of medicine - Cairo University- Egypt) The animals were maintained in an air-conditioned room at $20-25^{\circ} \mathrm{C}$, with a 12 -h light-dark cycle, and acclimated for 3-4 days before starting the experiments ${ }^{[17]}$. Then the rats were assigned to 4 groups $(n=10)$ and fed with the different diets for four weeks. Normal diet (ND), rats fed with the basic diet; high fat diet (HFD); CHP and CHNPs groups, rats fed with high fatty diet and administered orally water suspension of either chitosan or CHNPs, respectively (450 $\mathrm{mg} / \mathrm{kg} /$ day). At the end of the experimental period, blood samples were withdrawn from the orbital venous plexus using a capillary tube under ether anesthesia after an overnight fast. All animals have received human care in compliance with the guidelines of the Animal Care and Use Committee of the Pharmacology and Chemistry Research Centre (6th October City, Giza, Egypt).

\section{Biochemical analysis}

Serum was separated via centrifugation at $3000 \mathrm{rpm}$ for five minutes, then TC, TG, HDL-C, were colorimetric measured according to kit protocols [18,19] using (UV/VIS spectrophotometer, Shimadzu UV1800). LDL was calculated using the equation ${ }^{[20]}$

$$
\text { LDL-C }(\text { in } \mathrm{mg} / \mathrm{dl})=\mathrm{TC}-(\mathrm{HDL}-\mathrm{C}+\mathrm{TG} / 5)
$$

\section{Statistical analysis}

All data were expressed as means + SD. Differences between the groups were determined by one-way analysis of variance, using a statistical analysis software program (SPSS for windows, version Rel, 16.0, Spss Inc, Chicago, IL); $\mathrm{p}<0.05$ was considered to indicate statistical significance.

\section{Results and discussion}

\section{Characterization of chitosan}

The FTIR spectrum (vmax $\mathrm{cm}^{-1}$ ) of chitosan shows the characteristic bands of chitosan at $3440 \mathrm{~cm}^{-1}$ (broad) for $\mathrm{NH}$, and $\mathrm{OH}$ groups, $2915 \mathrm{~cm}^{-1}$ for $\mathrm{C}-\mathrm{H}$ stretching vibration and $1640 \mathrm{~cm}^{-1}$ (amide) (data not shown). TEM image (Fig 1) shows that, the chitosan nanoparticles were nearly spherical in shapewith external smooth surfaces, the average particles size was varying from 20$60 \mathrm{~nm}$. The stored chitosan nanoparticles showed no detectable changes in color or odor and no visible microbial growth.

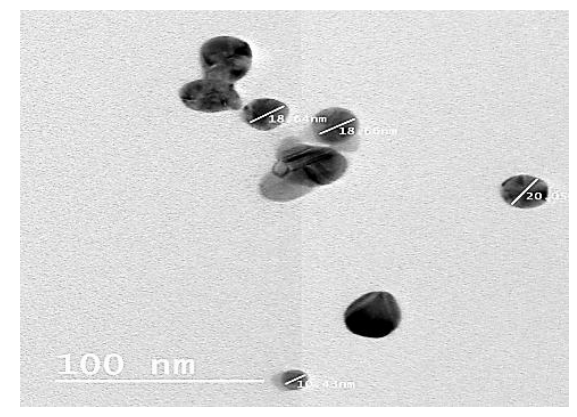

Fig (1): TEM image of chitosan nanoparticles

\section{General finding}

In contrast to the previous studies, we have prepared the fatty diet with the cooking oil and animal fats without addition of any other chemicals such as propylthiouracil, cholesterol, tween and sodium deoxycholate. Our diet simulates the conditions that caused by fast food consumption. So, our study may introduce more realistic data. Also, we have used a single daily dose that reported as recommended dose and didn't show obvious side effects ${ }^{[21]}$.

During all experimental time, all rats survived without signs of illness. There was no significant difference between control and treatment groups regarding physical activity, food and water intakes. Which may indicate that the chitosan administration has no obvious side effect and unrelated to appetite suppression.

\section{Effect on body weight}

Previous studies revealed that the consumption of chitosan had a beneficial lowering effect on body weight both in animals and humans ${ }^{[\mathbf{1 0 - 1 5}]}$. In our study, the body weight gain of HFD group showed significant increase compared with control group $(p<0.05)$. The feeding of chitosan in groups (CHP or CHNPs) showed a marked lowering effect on the body weight gain with no significant differences in average weight gain between the two groups (Fig 2). However, the weight gain was higher than the ND group, the results revealed the probability of using chitosan as weight controlling agent without life style change or diet control, taking in consideration the other safety parameters of possible side effects. 


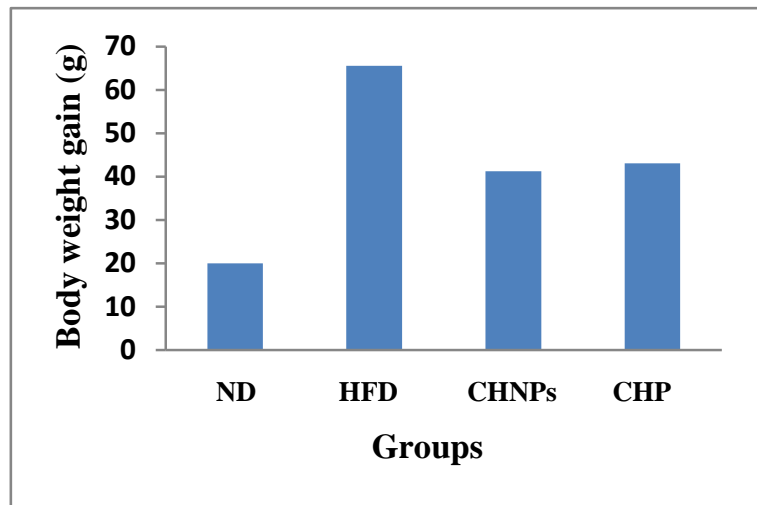

Fig (2): Effect of chitosan administration on body weight gain in rats

\section{Effect on serum lipids level}

When ingested, chitosan develops an HCl-layer in the stomach. As chitosan move through the duodenum, the $\mathrm{HCl}$-layer becomes diluted and the chitosan particles form agglomerates with fatty acids and cholesterol, thus reducing lipid absorption from the gastrointestinal tract [13].

As indicated from lipids analysis, the dyslipidemia was successfully induced in the HFD rats. Compared with control group, the serum concentrations of TC, TG, and LDL-C were significantly increased $(\mathrm{p}<0.05)$ while HDL-C level deceased as shown in (Fig 3, 4 \& 5). In CHP and CHNPS Groups, the feeding with chitosan significantly affects the blood lipids. As shown in fig (3) the TC levels were significantly decreased $(P<0.05)$ to retain the normal levels in both CHP and CHNPs groups without significant difference between the two groups. On the other hand, CHNPs showed more powerful effect than CHP to remove TG from the diet. However the TG levels in both groups remains in higher value than the normal one (Fig 4). As previously explained ${ }^{[22]}$, TG is an electrically neutral lipid molecule, so the positive charge of chitosan could not interact with the neutral TG molecule of the diet and then chitosan not able to remove all excess TG.

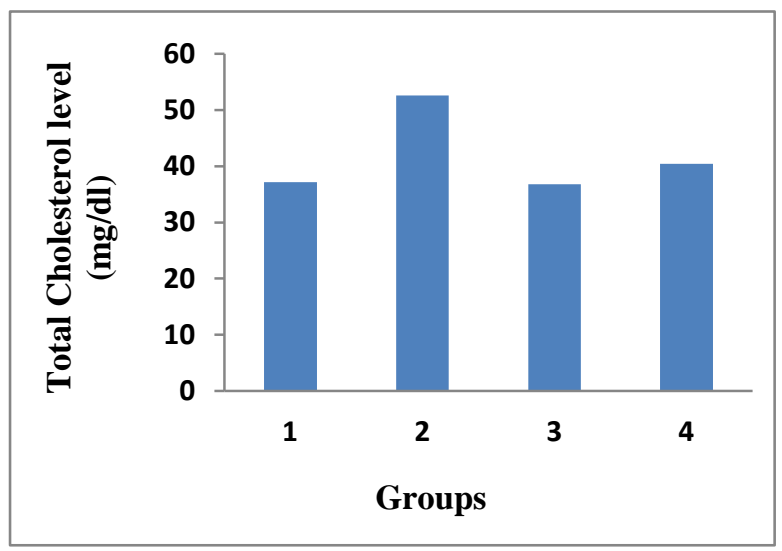

Fig (3): Effect of chitosan administration on total cholesterol level in rats

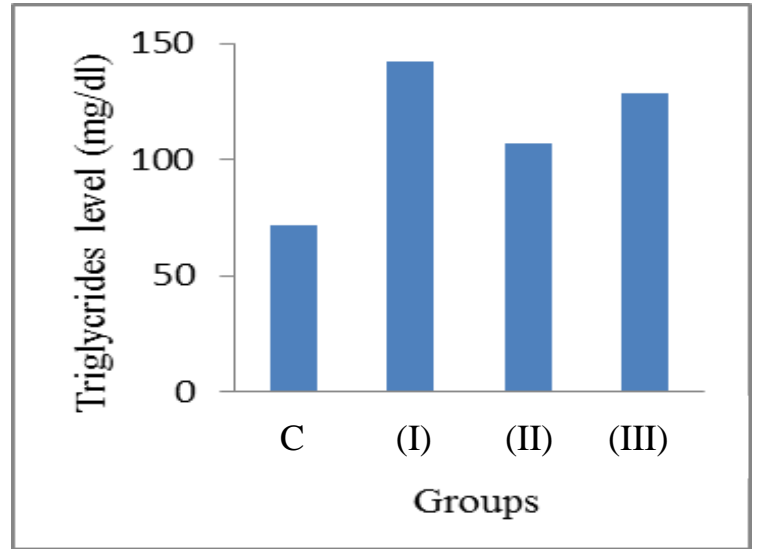

Fig (4): Effect of chitosan administration on triglycerides level in rats

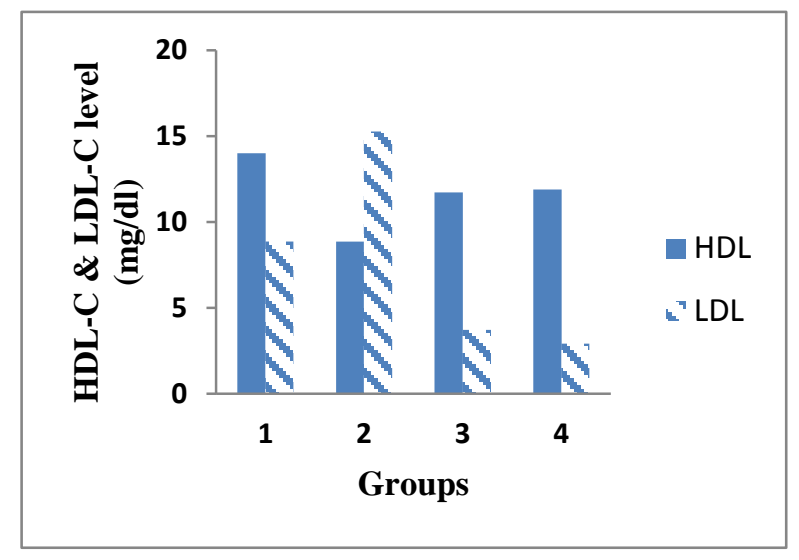

Fig (5): Effect of chitosan administration on HDL-C and LDL-C levels in rats

Finally, The HFD rats suffered from low levels of HDL$\mathrm{C}$ and the high levels of LDL-C that indicates an imbalance between cholesterol transportation from the liver to the extrahepatic tissues and back to the liver ${ }^{[11]}$. Feeding with chitosan significantly improved the HDL-C and LDL-C levels without significant difference between the two groups. In addition, the LDL-C level in both chitosan feeding groups was lower than the control group, which adds a new advantage of chitosan administration. All results are summarized in Table (1).

\section{Conclusion:}

Dyslipidemia has been successfully established in rats by high fat diet. Chitosan and chitosan nanoparticles administration was effective in lowering body weight gain and serum lipid levels in rats. In contrast to the previous study ${ }^{[4,11]}$, particle size effect was minimal and unlikely to be of statistically significance. Although CTNPs have the bigger surface area, CHP is characterized with higher porous structure. So the semisimilarity of lipid adsorption capacity of both size of chitosan may suggest that the interaction between chitosan and lipids is adsorption and entrapment, and the pore structure of chitosan contributes to its hypolipidemic effect. 
Table (1): Effect of chitosan and chitosan nanoparticles administration on body weight and serum lipids in rats.

\begin{tabular}{|c|c|c|c|c|}
\hline Parameter & ND & HFD & CHNPs & CHP \\
\hline Body weight gain (g) & $25.00 \pm 7.00$ & $62.00 \pm 11.00^{*}$ & $35.00 \pm 8.00^{\ddagger}$ & $37.00 \pm 6.00$ \\
\hline Total cholesterol (mg/dl) & $37.12 \pm 2.45$ & $52.59 \pm 2.98^{*}$ & $36.75 \pm 3.11^{\ddagger}$ & $40.44 \pm 2.47^{\ddagger}$ \\
\hline Triglycerides (mg/dl) & $71.28 \pm 6.25$ & $142.42 \pm 12.77^{*}$ & $106.71 \pm 11.85^{*}$ & $128.28 \pm 10.63$ \\
\hline HDL (mg/dl) & $14.00 \pm 1.24$ & $8.85 \pm 1.57^{*}$ & $11.71 \pm 1.78$ & $11.89 \pm 1.33^{\ddagger}$ \\
\hline LDL (mg/dl) & $8.86 \pm 0.98$ & $15.26 \pm 0.81^{*}$ & $3.7 \pm 0.64^{\dagger}$ & $2.9 \pm 0.33^{\ddagger}$ \\
\hline
\end{tabular}

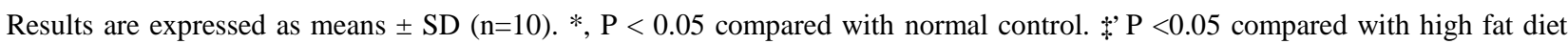
group.

\section{References}

1) Afshan, A., Salgar, V. B., Sugoor, M. and Deshpand, A. (2014). The impact of fast food consumption on the lipid profile, BMI and blood sugar levels. Int. J. Bioassays, 3(8):3221-3223.

2) Navar-Boggan, A. M., Peterson, E. D., D’Agostino R. B., Neely, B., Sniderman, A. D. and Pencina, M. J. (2015). Hyperlipidemia in early adulthood increases long-term risk of coronary heart disease. Circulation, 131:451-458.

3) Deepa, P. R. and Varalakshmi, P. (2005). Atheroprotective effect of exogenous heparinderivative treatment on the aortic disturbances and lipoprotein oxidation in hypercholesterolemic diet fed rats. Clin. Chim. Acta. 355:119-130.

4) Zhang, H. L., Taok, Y., Guo, J., Hu, Y. M. and Su, Z. Q. (2011). Hypolipidemic effects of chitosan nanoparticles in hyperlipidemia rats induced by high fat diet. Int. Immunopharmacol. 11:457-461.

5) Haitao, P., Qingyun, Y., Guidong, H., Chen, D., Peiqiu, C., Lanlan, H., Tiancun, X., Jiao, G. and Zhengquan, S. (2016). Hypolipidemic effects of chitosan and its derivatives in hyperlipidemic rats induced by a high-fat diet. Food \& Nutrition Research, 60:31137-31148.

6) Cheung, R. C., Ng, T. B., Wong, J. H. and Chan, W. Y. (2015). Chitosan: an update on potential biomedical and pharmaceutical applications. Mar. Drugs, 13:5156-5186.

7) Kumar, M. N., Muzzarelli, R. A., Muzzarelli, C., Sashiwa, H. and Domb, A. J. (2004). Chitosan chemistry and pharmaceutical perspectives. Chem. Rev., 104:6017-6084.

8) Martins, A. F., Facchi, S. P., Follmann, H. D.; Pereira, A. G.; Rubira, A.F.; Muniz, E. C. (2014), Antimicrobial activity of chitosan derivatives containing N-quaternized moieties in its backbone: a review. Int. J. Mol. Sci., 15:20800-20832.

9) Park, G. Y., Mun, S. and Park, Y. (2007). Influence of encapsulation of emulsified lipids with chitosan on their in vivo digestibility, Food Chemistry, 104(2):761-767.

10) Zhang, J., Liu, J., Li, L. and Xia, W. (2008). Dietary chitosan improves hypercholesterolemia in rats fed high-fat diets, Nutrition Research, 28(6): 383390.

11) Tao, Y., Zhang, H., Hu Y., Wan S. and Su, Z. (2013). Preparation of Chitosan and Water-Soluble Chitosan Microspheres via Spray-Drying Method to Lower Blood Lipids in Rats Fed with High-Fat Diets. Int. J. Mol. Sci., 14:4174-4184.

12) Qujeq, D. and Ataei, G. (2000). Effects of dietary chitosan on serum lipid and lipoprotein concentrations in rats. Iranian biomed. J., 4(2 \& 3):69-73.

13) Bokura, H. and Kobayashi, S. (2003). Chitosan decreases total cholesterol in women: a randomized, double-blind, placebo-controlled trial European J. Clinical Nutrition, 57:721-725.

14) Pittler, M. H. Abbot, N. C. Harkness, E. F. and Ernst, E. (1999). Randomized, double-blind trial of chitosan for body weight reduction. Eur. J. Clin. Nutr., 53:379-381.

15) Veneroni, G., Veneroni, F., Contos, S., Tripod, S., De Bernardi, M. and Guarino, C. (1996). Effect of a new chitosan dietary integrator and hypocaloric diet on hyperlipidemia and overweight in obese patients. ActaToxicolTher., 17:53-70.

16) Abd-Elhakeem, M. A., Ramadan, M. M. and Basaad, F. S. (2015). Removing of heavy metals from water by chitosan nanoparticles. J. advances in chemistry, 11(7):3765-3771.

17) Guide to the care and use of experimental animals (1993), Canadian council on animal care (CCAC), Canada, 1 (2nd edition).

18) Liu S. H., He S. P. and Chiang M. T. (2012). Effects of long-term feeding of chitosan on postprandial lipid responses and lipid metabolism in a high-sucrose-dietimpaired glucose-tolerant rat model. J. Agric. Food Chem., 60(17):4306-4313. 
19) Cui, X. B., Luan J. N., Ye, J. and Chen S. Y. (2015). RGC32 deficiency protects against high-fat diet-induced obesity and insulin resistance in mice. J. endocrinol., 224(2):127-137.

20) Nauck, M., Warnick, G. R. and Rifai, N. (2002). Methods for measurement of LDL-Cholesterol: A critical assessment of direct measurement by homogeneous assays versus calculation. Clin. Chem., 48(2):236-254.
21) Pittler, M. H., Abbot, N. C., Harkness, E. F. and Ernst, E. (1999). Randomized, double-blind trial of chitosan for body weight reduction. Eur. J. Clin. Nutr., 53:379-381.

22) Hossain, S., Rahman, A., Kabir, Y., Shamas, A. A., Afros, F. and Hashimoto, M. (2007). Effects of shrimp (Macrobraciumrosenbergii)-derived chitosan on plasma lipid profile and liver lipid peroxide levels in normal and hypercholesterolaemic rats. Clin. Exp. Pharmacol. Physiol., 34:170-176. 